\title{
Pour mieux franchir vos obstacles d'information : le Centre de documentation pour le sport
}

Easy Access Over the Information Hurdles Provided by the Sport Information Resource Centre

\section{Para mejor salvar sus problemas de información: el Centro de documentación para el deporte}

\section{Céline Gendron}

Volume 37, numéro 3, juillet-septembre 1991

URI : https://id.erudit.org/iderudit/1028474ar

DOI : https://doi.org/10.7202/1028474ar

Aller au sommaire du numéro

Éditeur(s)

Association pour l'avancement des sciences et des techniques de la documentation (ASTED)

ISSN

0315-2340 (imprimé)

2291-8949 (numérique)

Découvrir la revue

Citer cet article

Gendron, C. (1991). Pour mieux franchir vos obstacles d'information : le Centre de documentation pour le sport. Documentation et bibliothèques, 37(3), 99-105. https://doi.org/10.7202/1028474ar
Résumé de l'article

Le Centre de documentation pour le sport (CDS) possède une bibliothèque d'information sportive qui répond aux besoins spécifiques des entraîneurs, des étudiants en éducation physique, des administrateurs de sports, des professionnels de santé physique ou de médecine sportive, des psychologues ainsi que des amateurs de sports. C'est aussi une base de données disponible sur les grands serveurs internationaux doublée d'un accès sur CD-ROM. Le CDS publie également divers outils bibliographiques sur le sport et la condition physique. Il est particulièrement actif sur la scène internationale comme membre de l'International Association for Sports Information (IASI) et ses expériences de partenariat font partie intégrante de sa mission depuis ses débuts.
Tous droits réservés (C) Association pour l'avancement des sciences et des techniques de la documentation (ASTED), 1991
Ce document est protégé par la loi sur le droit d'auteur. L'utilisation des services d'Érudit (y compris la reproduction) est assujettie à sa politique d'utilisation que vous pouvez consulter en ligne.

https://apropos.erudit.org/fr/usagers/politique-dutilisation/ 


\title{
Pour mieux franchir vos obstacles d'information: le Centre de documentation pour le sport
}

\author{
Céline Gendron \\ Centre de documentation pour le sport \\ Gloucester, Ontario
}

Le Centre de documentation pour le sport (CDS) possède une bibliothèque d'information sportive qui répond aux besoins spécifiques des entraîneurs, des étudiants en éducation physique, des administrateurs de sports, des professionnels de santé physique ou de médecine sportive, des psychologues ainsi que des amateurs de sports. C'est aussi une base de données disponible sur les grands serveurs internationaux doublée d'un accès sur CD-ROM. Le CDS publie également divers outils bibliographiques sur le sport et la condition physique. II est particulièrement actif sur la scène internationale comme membre de I'International Association for Sports Information (IASI) et ses expériences de partenariat font partie intégrante de sa mission depuis ses débuts.

\section{Easy Access Over the Information Hurdles Provided by the Sport Information Resource Centre}

The Sport Information Resource Centre (SIRC) houses a sports information library that meets the requirements of trainers, physical education students, sports administrators, health and sports medicine professionals, psychologists, and interested sports fans. It is also a data base available through international online vendors and on CD-ROM. The SIRC publishes several bibliographic guides to sports and physical fitness literature. The Centre is active on the international scene through its membership in the International Association for Sports Information (IASI). Furthermore, its partnership experiences have been firmly integrated into its corporate mission since its creation.

\section{Para mejor salvar sus problemas de información : el Centro de documentación para el deporte}

El Centro de documentación para el deporte comprende una biblioteca de información deportiva que responde a las necesidades especificas de los entrenadores, de los estudiantes en educación física, de los administradores de deportes, de los profesionales de la salud física o de la medicina deportiva, de los psicólogos y de los aficionados de deportes. Es también una base de datos disponible sobre los grandes sistemas informativos internacionales $y$ accesible sobre CD-ROM. Este Centro de documentación publica además diversas obras bibliográficas sobre el deporte y la condición física. Es particularmente activo en la escena internacional como miembro de la International Association for Sports Information (IASI) y sus experiencias de colaboración con otras organizaciones forman parte integrante de su misión desde sus principios.
Fondée en 1845, la petite municipalité doit son nom au roi Charles-Albert de Savoie. Bien entendu, Albertville n'est pas la station balnéaire la plus fréquentée! Et pour cause, puisque cette localité française sera I'hôte des XVIe Jeux Olympiques d'hiver en février 1992. En prévision de cet événement, des milliers d'athlètes à travers le monde se préparent et s'entraînent depuis plusieurs années pour avoir le privilège de monter sur le podium des médaillés; mais derrière eux se profilent des entraîneurs, des psychologues, des gestionnaires, des spécialistes de la médecine sportive, des journalistes et bien sûr des... parents. Ici même au Canada, le Centre de documentation pour le sport (CDS) joue aussi un rôle important dans la préparation de cette olympiade puisqu'il gère une collection unique de documents spécialisés sur le sport, l'éducation physique, la condition physique et la médecine sportive. Situé en banlieue d'Ottawa, le CDS se distingue dans le monde comme chef de file en matière d'information sur le sport.

\section{Historique}

L'idée d'un centre de documentation pour le sport fut formulée pour la première fois au sein de I'Association canadienne des entraîneurs (ACE) par Douglas Fisher, journaliste et I'un des fondateurs de I'ACE, Geoff Gowan, alors coordonnateur technique de I'ACE et aujourd'hui président de la même association et Roger Jackson, alors directeur du Plan des Jeux olympiques de 1976. En 1973, les membres du conseil d'administration de I'ACE chargeaient Martha Stone, alors directrice des bibliothèques à
Santé et Bien-être social Canada de mener une étude sur la nécessité de mettre sur pied un tel centre. Le rapport devait établir qu'on ne retrouvait nulle part au Canada un centre de ce genre qui soit satisfaisant. Même dans les universités ayant un programme d'éducation physique reconnu, les collections de livres étaient généralement peu complètes. Parallèlement, le fonds de la petite bibliothèque spécialisée consacrée à l'éducation physique à l'Université d'Ottawa allait être versé à la collection principale de la bibliothèque centrale de l'Université; la bibliothèque perdait ainsi son statut et son bibliothécaire spécialisés. Le CDS a été fondé en 1973 et compte aujourd'hui treize employés permanents qui se partagent les différentes fonctions inhérentes à la gestion d'une base de données et d'un centre de documentation (voir organigramme du CDS). 


\section{Documentation et bibliothèques}

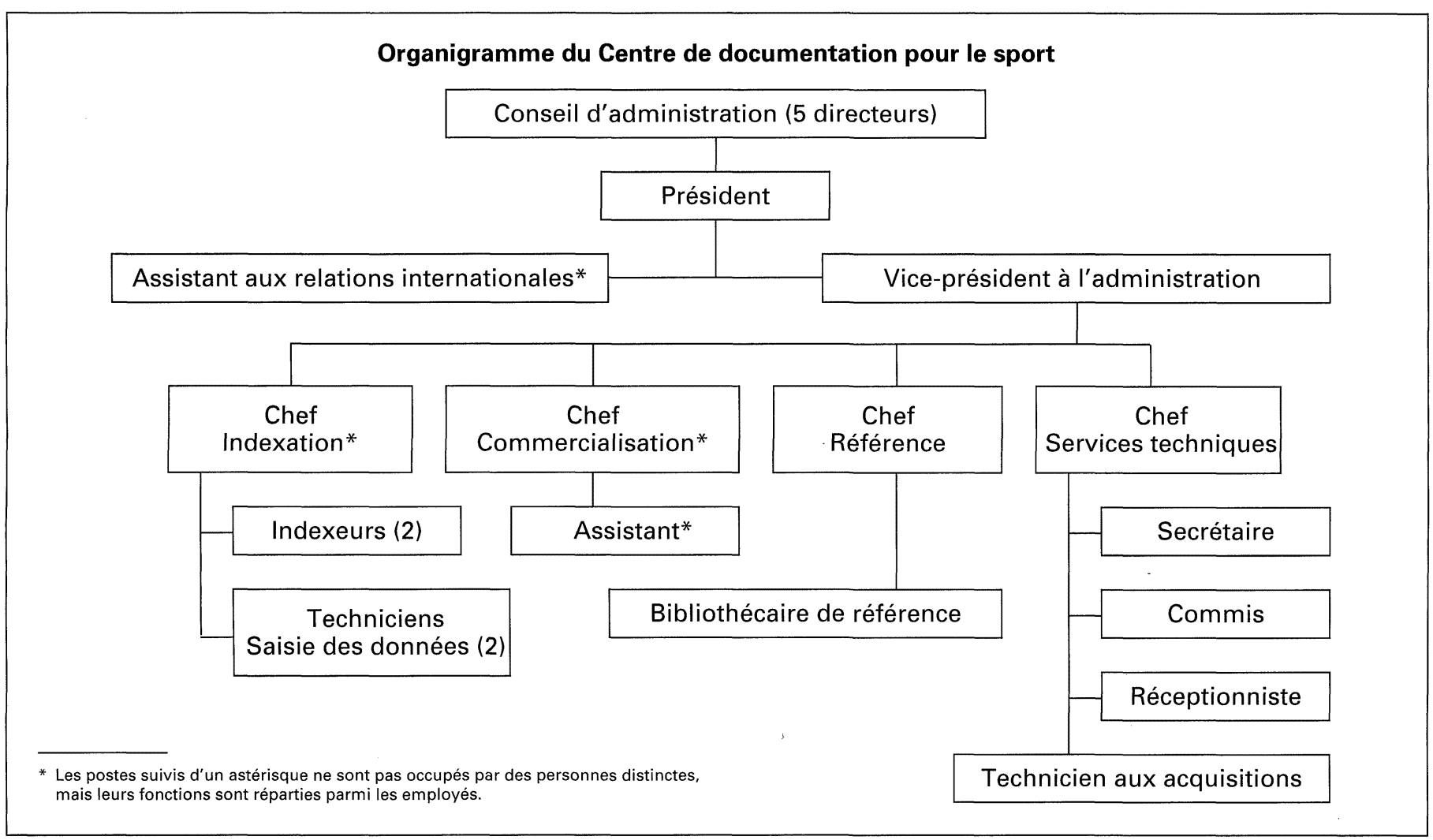

\section{Mandat du CDS}

À cette époque, la documentation sur le sport en Europe était assez élaborée. Après une visite auprès des principaux centres de documentation pour le sport en Angleterre, aux Pays-Bays, en France, en Belgique, en RFA, en Suisse, en Autriche et en Italie, le directeur du CDS se rendit compte que la RFA, et en particulier le Bundesinstitut fuer Sportwissenschaft (BISp), surpassait tout ce qui existait. Le BISp avait en effet élaboré une banque informatisée dans le cadre des Jeux de Munich de 1972. II s'agissait d'un système très avant-gardiste mais qui ne renfermait que de l'information hautement scientifique et souvent peu accessible aux entraîneurs canadiens. En outre, la banque de données du BISp couvrait de façon exhaustive les documents en allemand mais présentait de graves lacunes dans le cas des documents en langue anglaise et française. Le CDS avait le mandat de rassembler non seulement les ouvrages scientifiques mais aussi la documentation pour les entraîneurs désirant approfondir leurs connaissances et ce, dans les deux langues officielles. C'est dans cet esprit que les cadres de I'ACE décidèrent que le centre de documentation devait couvrir le domaine du sport en entier, en raison du fait que l'entraînement est un champ multidisciplinaire. Le CDS devenait donc une entité reliée et administrée par l'ACE jusqu'en 1985.

\section{Le système informatisé du CDS et la banque de données SPORT}

À la suite d'une étude faite en 1974 par la Digital Methods Ltd sur I'utilisation d'un système de récupération de l'information, le CDS a adopté le programme ISIS disponible par l'intermédiaire du Centre de recherches pour le développement international (CRDI). L'année suivante, le CDS créait une base de données qui, en 1982, sous le vocable de base de données SPORT, comptait 100000 notices (tableau 1). SPORT contient aujourd'hui plus de 275000 notices sur la littérature scientifique et technique internationale incluant la collection intégrale des microfiches de I'International Institute for Sport and Human Performance de l'Université d'Oregon. La base de données offre une documentation multidisciplinaire couvrant les domaines de la pratique, la stratégie, les techniques, les blessures, l'entraînement, l'équipement, la psychologie du sport, la médecine sportive, la biomécanique, la physiologie de l'exercice, la formation, l'arbitrage, les sports pour handicapés, les compétitions de tous niveaux et l'histoire du sport international. La base de données est officiellement reconnue comme étant un outil clé par I'International Association for Sports Information (IASI), le Conseil international pour l'éducation physique et la science du sport (CIEPSS) et I'Unesco.

En 1979, SPORT était relié à Infomart/SDC faisant de cette dernière la première base de données canadienne au contenu international reliée à un réseau international de banques de données. Toutes les bibliothèques canadiennes, ainsi que tous les pays couverts par le réseau de communication, pouvaient désormais accéder directement aux données analysées par le CDS.

En 1984, SPORT est devenu disponible sur CAN-OLE, le service canadien d'interrogation en direct de l'Institut canadien de l'information scientifique et technique (ICIST). D'autres serveurs ont suivi les traces de CAN-OLE: BRS (New York) en 1985, DIALOG en 1987 


\begin{tabular}{|c|c|}
\hline \multicolumn{2}{|c|}{ Tableau 1} \\
\hline \multicolumn{2}{|c|}{$\begin{array}{c}\text { Croissance de la base } \\
\text { de données SPORT } \\
\text { (du début au 1er juin 1991) }\end{array}$} \\
\hline & nombre de notices \\
\hline 1975 & -- \\
\hline 1982 & 100000 \\
\hline 1987 & 200000 \\
\hline 1991 & 275000 \\
\hline
\end{tabular}

(File 48), DIMDI (serveur allemand), de 1986 à juin 1991 puis DATASTAR (logé en Suisse) en 1990.

C'est en janvier 1989 qu'un tout nouveau marché s'est ouvert pour le CDS avec I'introduction du SPORT Discus, la version CD-ROM de la base de données, disponible par l'intermédiaire de SilverPlatter Information Inc. Les mises à jour se font deux fois par année et le logiciel d'interrogation SilverPlatter est compatible avec IBMPC et Apple de Macintosh (MacSPIRS). Une fonction d'aide à l'écran est disponible et des écrans "guide" en direct mettent en relief les caractéristiques de la base de données. À des fins comparatives, il suffit de se référer à la figure 1 qui présente des exemples d'une notice de la base de données SPORT selon les différents systèmes.

\section{Indexation}

Tous les documents de la base de données SPORT sont indexés par l'équipe professionnelle $d$ 'indexeurs du CDS. Chaque document est repérable au moyen de mots clés spécifiques au sujet de recherche. Afin de raffiner davantage les recherches dans la base de données, les documents sont indexés à trois niveaux : B - niveau élémentaire, I - niveau intermédiaire et $\mathbf{A}$ - niveau avancé. Dans le cas de documents de recherche, les références sont normalement accompagnées d'un résumé descriptif. Cette méthode de classification des documents s'avère très efficace pour les utilisateurs de la base de données et leur permet de trouver des réponses précises à leurs questions souvent complexes.

Avec les années, les indexeurs du CDS ont élaboré le SPORT Thesaurus qui contient plus de 8000 descripteurs et plus de 2000 renvois. L'édition de
1990 a été révisée de façon à refléter les développements récents dans les domaines de la médecine sportive, de la science du sport, de la condition physique et des disciplines connexes. Le Thesaurus comporte quatre sections: la première contient l'ensemble de la terminologie sportive et non sportive (médicale, scientifique, des sciences sociales). La deuxième englobe les termes géographiques. La troisième regroupe les équipes sportives, les associations et les clubs inclus dans la base de données. La q̊uatrième section comprend les codes bibliographiques qui servent de complément à l'utilisation des descripteurs dans la recherche bibliographique de la base de données SPORT. Le Thesaurus est disponible en accès direct chez Dialog et BRS. II est, de plus, inclus au SPORT Discus depuis juillet 1991.

Afin d'aider les utilisateurs de la base de données, l'équipe d'indexeurs du CDS a aussi conçu le SPORT Database User Aid. Ce document fournit des informations sur les pratiques $d$ 'indexation, la description des zones de la base de données, les formats $d^{\prime}$ affichage selon lẻs différents systèmes et la liste des périodiques indexés dans la base de données. Une nouvelle édition de cette publication est prévue pour 1992.

\section{La clientèle du CDS}

La clientèle desservie par le CDS rassemble le personnel des organismes nationaux de sport (ONS) ainsi que le personnel de Condition physique et Sport amateur au ministère de Santé et Bien-être social Canada. Pour illustrer les différentes composantes de sa clientèle, le CDS a schématisé, sous forme d'organigramme, la communauté sportive canadienne.

La Loi sur la santé et le sport amateur, promulguée en 1961, stipule que le mandat de Condition physique et Sport amateur est de "favoriser, promouvoir et développer la condition physique et le sport amateur au Canada $" 1$. Les deux principaux programmes, soit Condition physique Canada et Sport Canada, remplissent

1. Condition physique et Sport amateur, Condition physique et Sport amateur: Rapport annuel 1989-1990, Ottawa, Ministre des Approvisionnements et Services, 1991, p. 8.

\section{FIGURE 1}

\section{BRS (SFDB)}

AN ACCESSION NUMBER : 265234. 9012. AU AUTHOR: Fossil - K.

TI TITLE : Les Jeux d'hiver - une vitrine mondiale pour Lillehammer.

(The Winter Games - the world looks to Lillellammer.).

SO SOURCE : Olympic review (Lausanne, Switz.)(275/276) Sept/Oct 1990, 438-440. YR YEAR: 1990.

LG LANGUAGE : FR.

CC COUNTRY CODE : 756.

DE DESCRIPTORS : Olympic-Games-

Lillehammer-1994.

BB BIBLIOGRAPHY NUMBER : 287005. LV LEVEL: BASIC (B).

PT PUBLICATION TYPE : PERIODICAL ARTICLE (S).

OI ORDER INFORMATION : 265234.

\section{CAN/OLE (SPORT)}

AN - 1466525

RE - 265234

TI - Les Jeux d'hiver - une vitrine mondiale pour Lillehammer.

(The Winter Games - the world looks to Lillehammer.)

AU - Fossil, $\mathrm{K}$.

PU. - Olympic review (Lausanne, Switz.) (275/276), Sept/Oct 1990, 438-440

CP - Switzerland

LA - French

DT - Article ; Basic

CC - 287005; CC756

DE - Olympic Games, Lillehammer 1994

LO - SIRC article number: 265234 : Available from OOFS

\section{DIALOG (File 48)}

0265234

Les Jeux d'hiver - une vitrine mondiale pour Lillehammer.

The Winter Games - the world looks to Lillehammer.

Fossil, K.

Olympic review (Lausanne, Switz.), 275/276, Sept/Oct 1990, 438-440

LANGUAGE(S): French DOCUMENT TYPE: Journal article

COUNTRY OF PUBL. : Switzerland

LEVEL: Basic

SECTION HEADING : 287005 Olympic games - Lillehammer 1994 - Administration

KEYWORDS: Olympic Games, Lillehammer 1994

\section{SPORT Discus}

$\mathrm{TI}$ : Les Jeux d'hiver - une vitrine mondiale pour Lillehammer. (The Winter Games the world looks to Lillehammer.)

AU : Fossil,-K.

JN : Olympic review (Lausanne, Switz.) (275/276), Sept/Oct 1990, 438-440

PY : 1990

LA : French

DT : Serial-Article

LE : Basic

DE : Olympic-Games,-Lillehammer-1994

SH : (287005) OLYMPIC-GAMES-1994LILLEHAMMER-ADMINISTRATION

SC : 287005, 287

CP : Switzerland (756)

SF : SIRC

AN : 265234

UD : 9101 


\section{Schématisation de la clientèle primaire du CDS*}

Associations de sport

Condition physique et Sport amateur

Associations de loisirs

Organismes de soutien et spécialisés

Associations de sport pour handicapés

Associations des Jeux
Sport Canada

Condition physique Canada

Le CDS répond aussi aux demandes ponctuelles des organismes suivants: les fédérations des principaux Jeux, les fédérations sportives internationales et régionales, le Comité international olympique, les associations des comités olympiques nationaux, les Centres de haute performance, les fédérations sportives provinciales, les organismes sportifs provinciaux, les clubs, etc.

ce double rôle par leurs contributions financières aux ONS et aux associations axées sur la condition physique, aux agences, institutions, au secteur privé et aux gouvernements d'autres paliers associés à des projets relatifs au sport et à la condition physique.

Les organismes nationaux de sport (ONS) reconnus par le Gouvernement, et donc admissibles aux subventions, sont des organismes de sport amateur sans but lucratif qui représentent les intérêts des associations d'au moins huit provinces et territoires ${ }^{2}$. Les ONS sont composés de professionnels qui, par leurs compétences techniques et leur leadership, contribuent à l'élaboration de programmes récréatifs de grande qualité et de compétitions de haut calibre. Ces professionnels sont des directeurs techniques, directeurs de compétitions, spécialistes en marketing, entraîneurs, personnel de soutien, etc. Sur la centaine d'ONS associés au sport et à la condition physique, environ 65 résident au Centre canadien d'administration du sport et de la condition physique (CCASCP) à Ottawa ${ }^{3}$. Les autres associations sont réparties à travers le Canada. Le tableau 2 donne la répartition des ONS.

Dans cette optique, le CDS a conçu une trousse d'information pour le personnel des ONS et organise pour eux des visites personnalisées. L'état des collections reflète les besoins des organismes dans leur domaine spécifique mais aussi celui de la gestion de leur association. La participation du
CDS au réseau CAN/SDI a également permis de raffiner la diffusion sélective de l'information de la base de données SPORT auprès de la communauté sportive. Des profils d'intérêt sont également reçus de MEDLINE, ERIC, Compendex, Psychological Abstracts, etc. Les profils spécialisés permettent àinsi aux directeưrs techniques des associations sportives nationales, aux conseillers de Condition physique et Sport amateur et aux chercheurs qui travaillent en étroite collaboration avec. Sport Canada de se tenir au courant des dernières publications. Le service de reproduction d'articles du CDS répond ensuite aux demandes issues des profils reçus par ses usagers. Les demandes de prêt de documents et celles de prêt entre bibliothèques sont acheminées au service technique. Le CDS a développé une collection imposante et complètement automatisée de dossiers éphémères. II est donc possible de retracer les coupures de journaux, brochures et autres types de documents en repérant ceux-ci avec I'aide d'une liste de quelque $\mathbf{2 3 0}$ mots utilisés pour indexer ces documents. Le tableau 3 donne un aperçu de la collection et des activités du centre en 1990-1991.

C'est avec l'implantation, en 1985, du Programme de développement professionnel pour les directeurs $\mathrm{d}^{\prime}$ association (PDPDA) que le CDS a mis l'accent sur l'élaboration d'une toute nouvelle collection spécialisée de documents en gestion. Depuis, la collection s'est enrichie considérablement et le CDS a publié en 1991 un
Tableau 2

Répartition des associations résidentes et non résidentes au CCASCP*

\begin{tabular}{|ccc|}
\hline ONS & $\begin{array}{c}\text { rési- } \\
\text { dentes }\end{array}$ & $\begin{array}{c}\text { non } \\
\text { résidentes }\end{array}$ \\
\hline $\begin{array}{l}\text { Associations } \\
\text { de sport }\end{array}$ & $51 / 79$ & $28 / 79$ \\
$\begin{array}{l}\text { Associations } \\
\text { de loisir }\end{array}$ & $3 / 9$ & $6 / 9$ \\
$\begin{array}{l}\text { Organismes } \\
\text { de soutien et } \\
\text { spécialisés }\end{array}$ & $11 / 16$ & $5 / 16$ \\
$\begin{array}{l}\text { Associations de } \\
\text { sport pour } \\
\text { handicapés }\end{array}$ & $3 / 7$ & \\
$\begin{array}{l}\text { Associations } \\
\text { des Jeux }\end{array}$ & $3 / 6$ & $3 / 7$ \\
$\begin{array}{l}\text { * Source d'information: Sports Federation of } \\
\text { Canada/La Fédération des sports du Canada, } 1991 \\
\text { Directory. Répertoire, Sports Federation of Canada/ } \\
\text { La Fédération des sports du Canada, Gloucester, } \\
\text { Ont., 1991, 193 p. }\end{array}$ \\
\hline
\end{tabular}

outil de référence intitulé Ressources de gestion destiné principalement au personnel des associations sportives.

\section{Publications}

En août 1974, le CDS publiait le premier numéro de ce qui était alors appelé Articles de revues sportives et qui recensait les articles des derniers périodiques reçus. Après quelques transformations, cette publication est devenue SportSearch qui se veut avant tout un outil de sensibilisation à l'actualité sportive. Elle dépouille mensuellement plus de 1200 périodiques français ou anglais; on y retrouve aussi des monographies, des articles pertinents tirés de périodiques autres que ceux spécialisés dans le sport de même que des chapitres de livres et

2. Les ONS sont de création relativement récente telle I'Association canadienne de softball amateur (1972) ou peuvent exister depuis des décennies comme dans le cas de l'Association canadienne de patinage de vitesse amateur (1887). La visibilité, le budget et le personnel des ONS varient considérablement d'une association à l'autre: par exemple, l'Association canadienne de hockey amateur a reçu un budget de 1299000 \$ en 89-90 et dispose d'un personnel permanent de 26 employés; I'Association canadienne de handball a reçu $46000 \$$ et elle compte trois employés permanents.

3. Le CCASCP loge également le Centre de documentation pour le sport. 


\section{Tableau 3}

\section{État des collections du CDS et des activités des Services techniques en 1990-1991}

Monographies

Thèses

Périodiques

Articles de périodiques

Profils d'intérêt

Dossiers éphémères

Articles photocopiés

Prêts

PEB
38965 indexées dans SPORT dont

29055 se trouvent dans la collection

10731 sur microfiches indexées dans SPORT dont 8663 se trouvent dans la collection

1448 titres reçus couramment

8287 numéros reçus en $89-90$

192630 indexés dans SPORT dont 166493 se trouvent dans la collection

\section{1}

2625 indexés dans SPORT

6380

1004

868 (prêtés)

1256 (empruntés) des comptes rendus de conférences. Des vedettes-matière renvoient directement au sujet et une liste d'éditeurs et adresses des périodiques dépouillés se retrouve en annexe afin de faciliter l'abonnement aux périodiques mentionnés. SportSearch contient aussi une liste des conférences scientifiques et professionnelles reliées au domaine du sport et de la condition physique. Tous les organismes responsables de ces conférences sont régulièrement sollicités afin d'alimenter la base de données du CDS des comptes rendus de leurs travaux et délibérations.

En 1981-1982, le CDS publiait la bibliographie la plus importante et la plus complète jamais inventoriée sur le sport. Elle comporte huit volumes donnant plus de 70000 références. Une mise à jour présentée en deux volumes comprenant 30000 références est venue la compléter en 1983. Le CDS a décidé de mettre un terme à la publication en 1987, après la parution de trois volumes supplémentaires. L'accès rapide et direct à la base de données sur plusieurs systèmes, la manipulation plutôt laborieuse de bibliographies imprimées et l'aug= mentation de coûts de production justifiaient une telle décision. Afin de répondre davantage aux besoins de la communauté sportive et aux demandes sans cesse croissantes de divers intervenants, le CDS a inauguré en 1990 une nouvelle collection, Sport-
Biblio, qui vise à cerner davantage l'information sur un sujet précis. Chacun des numéros de la série porte sur un thème $d^{\prime}$ actualité et contient au-delà de 200 références bibliographiques. Une douzaine de titres sont déjà parus dont Éthique du sport, Commercialisation du sport amateur, Condition physique au travail, etc. En 1990, le CDS publiait la troisième édition de Sport et Loisirs pour Handicapés: une bibliographie 1984-1989. Avec plus de 4000 notices bibliographiques publiées dans des périodiques, des monographies, des thèses, des comptes rendus de conférences, etc., la bibliographie souligne de façon éclatante la part importante des recherches et activités entreprises dans ce secteur.

En juillet 1991, le CDS a publié Dossier dopage. Une bibliographie sur les drogues et le dopage dans le sport. Cette bibliographie servira de jalon au travail qu'effectuera le CDS en collaboration avec le Programme antidopage Canada.

\section{Division internationale}

Le CDS participe activement aux réunions de l'International Association for Sports Information (IASI). Fondée en 1960, I'IASI encourage la diffusion de l'information et de la documentation en sport et condition physique.
Pour ce faire, elle s'appuie sur les principes élaborés par I'UNICIST. L'association coordonne des projets d'intérêt général et apporte son expertise aux organismes qui se chargent de les réaliser. Le président du CDS est d'ailleurs président de I'IASI depuis mai 1989. En tant que chef de file reconnu, le CDS a souvent l'occasion de discuter des besoins de nouveaux centres de documentation sur les plans méthodologique et technique, ce qui tend à favoriser une meilleure normalisation des échanges internationaux d'information dans le domaine du sport.

C'est à la suite de sa participation à la réunion du Comité de coordination (COCO) de I'IASI en 1985, au cours de laquelle furent discutés différents aspects du développement d'une banque de données internationale, que le CDS a rédigé plusieurs principes directeurs: International SPORT Database Indexing Manual: Field Descriptions and Procedures Outline (juin 1985), Article Selection Policy for the SPORT Database (édition révisée en octobre 1985) et International SPORT Database: Indexing Examples (mars 1986) ${ }^{4}$. Dans le but d'alimenter la base de données, des ententes ont alors été conclues avec les pays suivants: I'Australie, le Brésil, la République fédérale allemande, Israël, les PaysBas et la Suisse. En 1991, plus d'une vingtaine de pays avaient signé un protocole d'accord avec le CDS afin de fournir des données à la base SPORT. Le CDS reçoit également des stagiaires en provenance d'autres pays qui s'intéressent à ses méthodes d'indexation. De retour dans leur pays, ces stagiaires sont souvent chargés de collaborer à la base de données SPORT en complétant des fiches d'indexation du CDS.

\section{Partenariat}

Depuis plusieurs années, le CDS est associé à de grands projets bibliographiques. En 1987, dans le cadre d'un projet spécial de bibliographie sur les femmes et le sport, projet subventionné et dirigé par le Programme des femmes pour le leadership sportif de Sport Canada, le CDS a siégé au sein

4. Ces principes directeurs ont été regroupés dans Scientific SportDatabase: Technical Manual (October 1986). 


\begin{tabular}{|c|c|c|}
\hline \multicolumn{3}{|c|}{$\begin{array}{c}\text { Tableau } 4 \\
\text { Contributions internationales } \\
\text { à la base de données SPORT }\end{array}$} \\
\hline & $\begin{array}{l}\text { nombre } \\
\text { de pays }\end{array}$ & $\begin{array}{c}\text { nombre } \\
\text { de notices }\end{array}$ \\
\hline 1986-1987 & 8 & 921 \\
\hline $1987-1988$ & 15 & 1323 \\
\hline 1988-1989 & 13 & 2398 \\
\hline $1989-1990$ & 15 & 3340 \\
\hline $1990-1991$ & 11 & 4713 \\
\hline
\end{tabular}

du Comité consultatif de ce Programme. Toujours en 1987, le CDS a produit une bibliographie sur les drogues et le dopage qui fut distribuée à I'IAF5 World Symposium on Doping in Sport. Une seconde bibliographie fut produite l'année suivante à I'occasion de la tenue au Canada de la Première Conférence mondiale permanente sur l'antidopage dans le sport.

À la suite des XVe Jeux Olympiques d'hiver à Calgary en 1988, un projet fut mis sur pied par Gretchen Ghent de l'Université de Calgary afin d'établir une bibliographie exhaustive regroupant les articles, monographies et documents relatifs aux Jeux de Calgary. La bibliographie sera terminée en novembre 1991. Ce projet a été réalisé en collaboration avec le CDS; ainsi toutes les références du produit imprimé seront également repérables dans la base de données SPORT et des copies des documents seront disponibles par l'intermédiaire du CDS.

Le rôle du CDS comme diffuseur d'information sportive au Canada est de plus en plus reconnu au niveau national. C'est ainsi qu'une plus grande concertation se développe parmi les organismes générateurs d'information afin d'éviter toute duplication au plan des ressources à produire et à diffuser. À titre d'exemple, le CDS collabore avec le Groupe de travail sur la politique fédérale en matière de sport. Ce groupe a été mis sur pied par le Ministre de Condition physique et Sport amateur en janvier dernier. Le Programme antidopage du Canada reconnaît aussi cette participation dans son plan organisationnel. II y fait mention de sa collaboration avec le CDS afin de développer une base d'information sur le dopage dans le sport.

Les organisations sportives internationales invitent quelquefois le CDS à les aider dans le plan de développement de leurs ressources d'information. L'International Rugby Football Board (IRFB) en est un exemple. Ce dernier subit présentement des changements structurels et organisationnels majeurs. Durant cette période, les responsables ont eu recours au CDS afin d'améliorer leurs ressources en information. Le Conseil a aussi entrepris des négociations avec le CDS afin que ce dernier devienne le Centre mondial d'information pour le rugby. Le IRFB a reconnu que l'information était vitale pour la réorganisation du rugby. II considère plus pratique de faire appel à l'expertise d'un leader déjà reconnu dans le domaine de l'information sportive plutôt que d'essayer d'établir son propre centre d'information. Une entente doit être conclue au début de l'année 1992.

L'exemple du International Indexing Cooperative Venture est un autre signe bien visible des expériences de partenariat initiées par le CDS. En 1986, huit pays avaient signé des ententes avec le CDS pour verser des notices de niveau intermédiaire et avancé à la base de données. Ils sont maintenant une vingtaine dont le National Sport Information Centre of the Australian Sports Commission avec plus de 6000 notices, I'International Institute for Sport and Human Performance de I'Université d'Oregon qui indexe et verse ses thèses sur microfiches et The Amateur Athletic Foundation, née des recettes engendrées par les Jeux de la XXIII Olympiade de Los Angeles en 1984. Le tableau 4 indique le nombre de notices dans la base de données SPORT provenant de contributions internationales.

\section{Projet avec les Affaires extérieures du Canada}

Déjà en 1987, le président du CDS proposait de créer au sein de I'IASI une commission ad hoc sur les besoins spécifiques des bibliothèques de sport des pays en voie de développement. La même année, le Secrétaire d'État aux Affaires extérieures du Canada, suite à une décision du Cabinet, annonçait l'intention de son ministère d'utiliser le sport comme instru-
Tableau 5

\section{Subventions reçues par Sport Canada}
1985-1986
$467000 \$$
1986-1987
$525457 \$$
1987-1988
$546315 \$$
1988-1989
$550550 \$$
1989-1990
$512000 \$$

ment de "diplomatie" culturelle. Parmi les éléments de la stratégie élaborée, l'idée de projets d'assistance au développement du sport fut retenue. $C^{\prime}$ est dans ce contexte que le CDS a présenté un projet de développement visant à utiliser ses trois ressources maximales, c'est-à-dire la base de données SPORT, une imposante collection de documents et son expertise en informatisation du travail et en traitement de documents. Le projet vise à fournir aux organismes de sport des pays en voie de développement l'information sportive nécessaire à la réalisation d'activités sportives et administratives. II a été réalisé avec I'aide des Relations internationales sportives du ministère des Affaires extérieures. Après consultation avec l'Association des comités nationaux olympiques d'Afrique (ACNOA), six pays ont été sélectionnés et six établissement retenus ${ }^{6}$. Ces derniers ont reçu du matériel informatique (logiciels et équipement pour CDROM) et ont bénéficié de sessions de formation. La deuxième phase du projet, c'est-à-dire le choix d'autres pays ou établissements, est présentement à l'étúde et les critères de sélection seront établis en fonction des résultats de la première expérience.

\section{Nouvelles réalités}

Fondé en 1973, le Centre de documentation pour le sport est devenu une société sans but lucratif indépendante

5. International Athletic Federation

6. Cameroun: Association des comités nationaux olympiques d'Afrique (ACNOA)

Égypte: Sport Research Institute, Giza University

Kenya: Kenya Olympic Association

Nigeria: Nigeria Olympic Committee

Zambie: National Olympic Committee

Zimbabwe: Zimbabwe Olympic Committee 


\section{Documentation et bibliothèques}

en avril 1985. Régi par un Conseil $\mathrm{d}^{\prime}$ administration, le CDS reçoit une subvention de Sport Canada équivalant à près de la moitié de son budget: le tableau 5 permet de visualiser la tendance budgétaire décroissante du CDS. Toutefois, ces subventions ont été plus ou moins gelées quand elles n'ont pas carrément diminué, au moment même où le Gouvernement encourage les organismes nationaux de sport (ONS) à trouver de nouvelles sources de financement. Pour les ONS, cela se traduit souvent par la recherche de sources externes de revenus tel le parrainage d'entreprise. Pour le CDS, la diminution de subventions exige de diversifier ses produits afin de générer davantage de revenus s'il veut maintenir ses opérations au même niveau de qualité. Le CDS y est parvenu en ciblant plus spécifiquement ses pro- duits et services à un point tel que ses revenus représentent maintenant $45 \%$ de son budget total alors qu'ils ne représentaient que $10 \%$ il y a cinq ans. Cela s'est réalisé dans un contexte de travail extrêmement stimulant puisque le personnel du CDS n'a pas vu ses effectifs augmenter. Afin de réaliser sa mission, le CDS s'est surpassé dans l'élaboration d'outils qui lui étaient nécessaires. C'est ainsi qu'au fil des ans, il a établi des moyens de sélectionner, emmagasiner, traiter et diffuser l'information sportive sur des supports variés toujours plus accessibles.

Par sa visibilité au sein de la communauté sportive canadienne et internationale et par l'expertise qu'il a su développer dans son créneau particulier, le CDS est en mesure de faire face aux divers obstacles qui se dressent: pénurie des ressources, compétitivité technologique, dépendance administrative et souvent sclérosée, etc. Cette situation dynamique repose en grande partie sur les expériences acquises, les échanges continus, l'ouverture et la disponibilité professionnelles. Car derrière la base de données, les produits et services et les échanges internationaux, il faut souligner la haute qualité des ressources humaines particulières au CDS. Ce sont elles qui matérialisent quotidiennement les réalisations du CDS et en font un centre unique sur le sport*.

* L'auteure remercie ses collègues du CDS pour leur collaboration et spécialement Gilles Chiasson, Jean Michel Johnson, Christine Lalande, Richard Stark et Linda Wheeler.

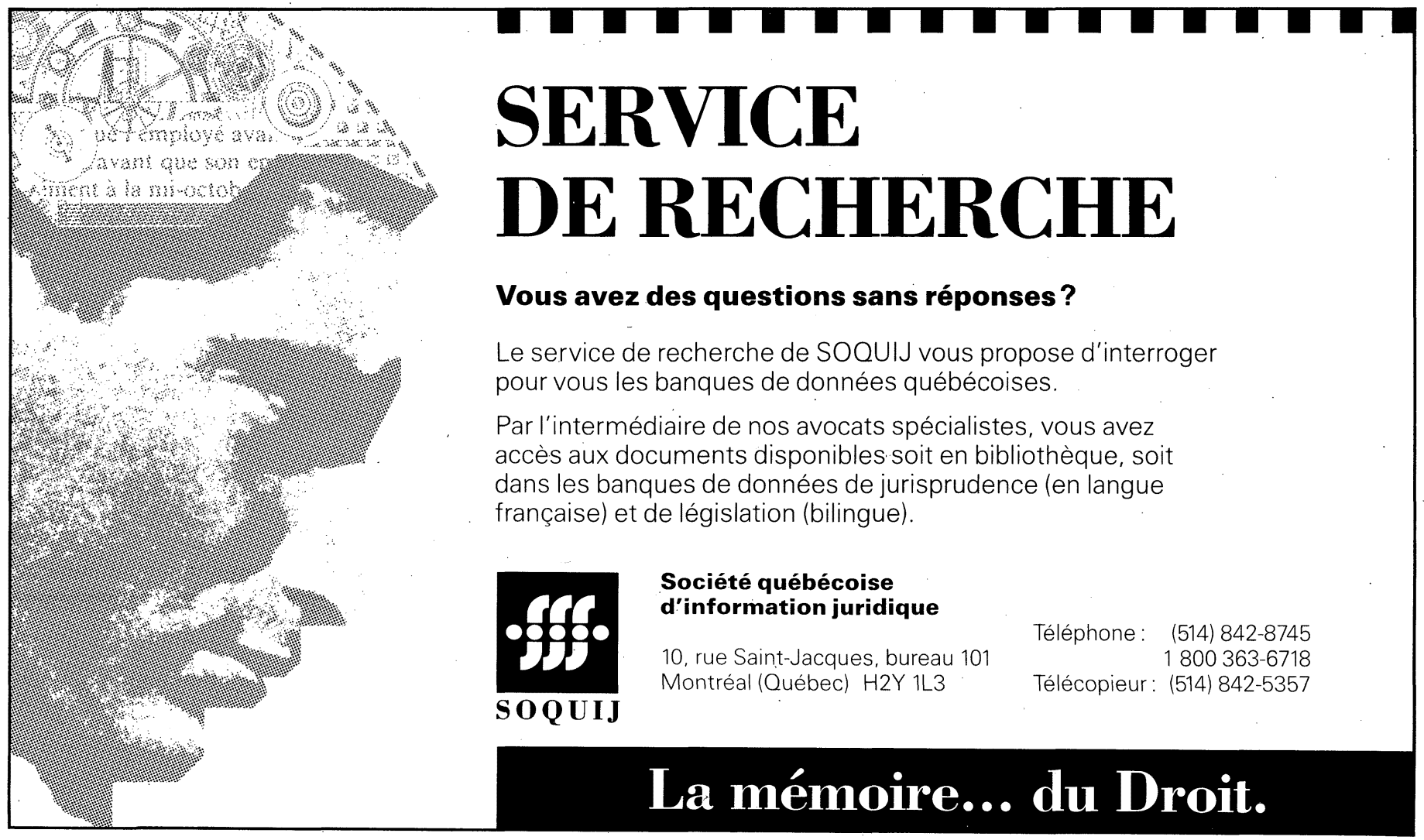

